\title{
QUEILOPLASTIA PRIMÁRIA UNILATERAL ATRAVÉS DA TÉCNICA DE FISHER
}

\section{UNILATERAL PRIMARY LIP REPAIR BY FISHER TECHNIQUE}

\author{
Pedro Henrique da Hora Sales* \\ Suellen Sombra da Rocha" \\ Assis Filipe Medeiros Albuquerque*** \\ José Ferreira da Cunha Filho"***
}

\begin{abstract}
RESUMO
Introdução: As fissuras labiais são alterações congênitas que decorrem da não fusão dos processos nasais medianos com os processos nasais laterais, sendo sua ocorrência em aproximadamente 1:550 nascidos vivos. Essas fissuras podem apresentar-se bilaterais ou unilaterais nos lábios e acompanhadas ou não de fenda palatina. A cirurgia primária para reparação do lábio fissurado unilateral vem sendo descrita por diversas técnicas na literatura e geralmente envolve rotações de retalho e deixam cicatrizes visíveis bem como encurtamento do vermelhão do lábio. A técnica de Fisher foi proposta para resultar em uma cicatriz menos visível, em linha reta na região do filtro labial, colocando-a em uma região mais anatômica e simplificando a cirurgia. O objetivo deste artigo é mostrar um caso clínico de queiloplastia primária unilateral, utilizando a técnica de Fisher. Relado de Caso: Paciente CDSS, 8 meses de idade, portador de fissura lábio-palatina unilateral do lado esquerdo. A cirurgia do lábio foi realizada em ambiente hospitalar sob anestesia geral e a técnica utilizada foi a de Fisher. No acompanhamento de 30 dias o paciente apresentou-se com uma cicatriz bastante discreta, posicionada simetricamente em relação ao filtro labial do lado oposto. Conclusão: A técnica de Fisher mostrase como ótima opção para o tratamento de fissuras labiais unilaterais, pois proporciona uma excelente estética, a técnica cirúrgica é relativamente simples e acompanhada de bons resultados funcionais.
\end{abstract}

Descritores: Fenda labial • Anormalidades congênitas • Doenças labiais.

\section{ABSTRACT}

Introduction: Cleft lip is a congenital abnormality arising from no merger of the median nasal processes with the lateral nasal processes, and their occurrence is approximately 1 in 550 live births. These clefts may present bilateral or unilateral lips and with or without cleft palate. The primary surgery for repair of unilateral cleft lip has been described by several techniques in literature and usually involves retail rotations and leave visible scars and lip vermilion shortening. Fisher technique has been proposed to result in a less visible scar, straight in the philtrum region placing it in a more anatomical region and simplifying the surgery. The purpose of this article is to show a case of unilateral primary lip repair using the Fisher technique. Case Report: Patient CDSS 8 months old has an unilateral cleft lip and palate on the left side. The lip surgery was performed in a hospital under general anesthesia and the technique used was Fisher. In the following 30 days, the patient is presented with a very slight scar, positioned symmetrically in relation to the philtrum of the opposite side. Conclusion: Fisher technique is shown as great option for the treatment of unilateral cleft lip, it provides an excellent aesthetic, the surgery technique is relatively simple and accompanied by good functional results.

Descriptors: Cleft Lip • Congenital abnormalities • Lip diseases.

\footnotetext{
* Residência em Cirurgia e Traumatologia Bucomaxilofacial pelo Hospital Instituto Dr. José Frota. Fortaleza-CE. salespedro@gmail.com

** Residente de Cirurgia e Traumatologia Bucomaxilofacial do Hospital Instituto Dr. José Frota. Fortaleza-CE.suellensombrarocha@gmail.com

*** Residência em Cirurgia e Traumatologia Buco-Maxilo-Facial - HUOL/UFRN. Especialista pelo CFO e Colégio Brasileiro de Cirurgia e Traumatologia Buco-Maxilo-Facial. Doutorando e Mestre em Odontologia (Área de Clínica Odontológica/Cirurgia Buco-Maxilo-Facial) - UFC. Professor do Curso de Odontologia do Centro Universitário Católica de Quixadá - UNICATÓLICA. Professor do Curso de Odontologia da Universidade de Fortaleza - UNIFOR. assis_filipe@hotmail.com

**** Mestre em Cirurgia pela Faculdade de Medicina da Universidade Federal do Ceará. Cirurgião Bucomaxilofacial do Hospital infantil Albert Sabin. jferreiracf@yahoo.com.br
} 


\section{N T R O D U Ç Ã O}

As fissuras lábio-palatinas são malformações congênitas que acometem o lábio superior e o palato. São ocasionadas por falhas no processo de fusão entre os processos nasais medianos e os processos nasais laterais e entre os processos maxilares, sendo sua etiologia incerta ${ }^{1}$. Sua incidência varia entre 1 para 550 a 1 para 1200 nascidos vivos, sendo mais prevalente no sexo masculino e em asiáticos ${ }^{1}$.

O período para realização da cirurgia de lábio é variável. Entretanto, a maioria dos autores concorda que a cirurgia deve ser realizada entre 3 e 6 meses de idade ${ }^{2}$. Questões como fatores socioeconômicos, culturais e até mesmo a grande procura nos serviços de saúde especializados fazem muitas vezes com que essas cirurgias sejam realizadas fora do tempo adequado.

Em geral, são cirurgias que apresentam poucas complicações, sendo as mais relatadas a dor pós-queiloplastia, seguida de taquicardia, estando esta mais associada à agitação pós-operatória ${ }^{3}$.

Durante o século XX, diversos autores propuseram técnicas para fechamento do lábio fissurado. As técnicas iniciais eram as chamadas técnicas em linha reta. Elas baseiam-se em incisões paralelas ao filtro labial, com a finalidade de colocar a cicatriz em uma posição anatômica mais favorável, entretanto apresentam como principal desvantagem o encurtamento do lábio que pode ocorrer pela retração cicatricial ${ }^{4,5}$.

Por conta disso as técnicas geométricas são hoje as mais utilizadas. Elas combinam rotações com avanços dos retalhos diminuindo consideravelmente a retração cicatricial por quebra na incisão reta. Alguns exemplos são as técnicas de Meara et al. ${ }^{5}$ (2011), Demke e Tatum ${ }^{6}$ (2011) e Fisher (2005) 7 .

A técnica de Fisher consiste em uma nova variação de técnica geométrica em que ele adiciona um pequeno triângulo inferior acima da linha muco-cutânea para rotação adicional caso seja necessário, preservando, dessa forma, as subunidades labiais e colocando a cicatriz em uma posição anatômica mais favorável, ou seja, paralela ao filtro labial ${ }^{1,7}$. O ob- jetivo deste estudo é relatar um caso clínico de queiloplastia unilateral utilizando a técnica de Fisher.

\section{RELATO DE CASO}

Paciente C.D.S.S., 8 meses de idade, compareceu com a mãe a um serviço de Cirurgia Bucomaxilofacial, referência na cidade de Fortaleza - Ceará, sendo portador de fissura lábio-palatina unilateral esquerda. Após anamnese e exame clínico foi proposto o tratamento cirúrgico através do fechamento primário da fissura labial pela técnica de Fisher.

A cirurgia foi realizada em ambiente hospitalar sob anestesia geral e intubação oro-traqueal. Foram realizadas marcações cutâneas com solução de Verde Brilhante, no filtro labial do lado direito, na região onde ficaria o filtro labial esquerdo e nos pontos entre a face interna da asa do nariz do lado esquerdo até um ponto de maior espessura do lábio superior do lado direito, na região da linha muco-cutânea. Esses dois últimos pontos devem ser marcados obedecendo o mesmo comprimento do filtro labial direito que é medido através de paquímetro no transoperatório e essa medida transferida para o lado esquerdo. Após marcação desses dois pontos, foi traçada uma linha entre eles para facilitar a visualização durante a cirurgia. É importante a manutenção dessa medida pois essa distância irá conferir simetria ao filtro labial e o correto posicionamento da cicatriz.

Após essa etapa foi realizada a marcação de um pequeno triângulo com ápice voltado para a fissura na região do lábio esquerdo. Esse triângulo irá permitir uma rotação de retalho para alongamento do filtro labial esquerdo caso seja necessário. E por fim marcações na linha muco-cutânea de ambos os lados da fissura devem ser realizadas até a mucosa labial (Figura 1a).

Foram realizadas incisões cutâneas nas regiões marcadas previamente e também incisões relaxantes, realizadas na mucosa labial por via intraoral a fim de diminuir a tensão e facilitar a coaptação dos bordos cruentos. A Musculatura do orbicular do lábio deve ser dissecada da pele do lábio e a sutura deve ocorrer inicialmente
SALES PHH

ROCHA SS

ALBUQUERQUE AFM

CUNHA-FILHO JF

QUEILOPLASTIA

PRIMÁRIA

UNILATERAL

ATRAVÉS DA

TÉCNICA DE

FISHER

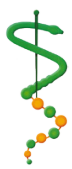

REV, ODONTOL.

UNIV, CID, SÃO

PAULO

2016; 28(2):

$148-54$, MA I - AGO 
SALES PHH

ROCHA SS ALBUQUERQUE AFM CUNHA-FILHO JF

QUEILOPLASTIA

PRIMÁRIA

UN I LATERAL ATRAVÉS DA TÉCNICA DE

FISHER

- 150 .

REV, ODONTOL :

UNIV, CID, SÃO

PAULO

$2016 ; 28(2):$ $148-54, M A I-A G O$ na musculatura (Figura $1 \mathrm{~b}$ ). Foi realizada também dissecção das cartilagens nasais através das incisões labiais pois essas se apresentam de maneira desorganizada no paciente com fissura lábio-palatina (Figura 1c). A sutura do músculo orbicular do lábio foi realizada com fio reabsorvível Vicryl 4-0, e posteriormente realizada a sutura da pele com fio monocryl 5-0, sendo o primeiro ponto na pele, realizado na região da linha muco-cutânea.

A mucosa labial foi suturada com fio

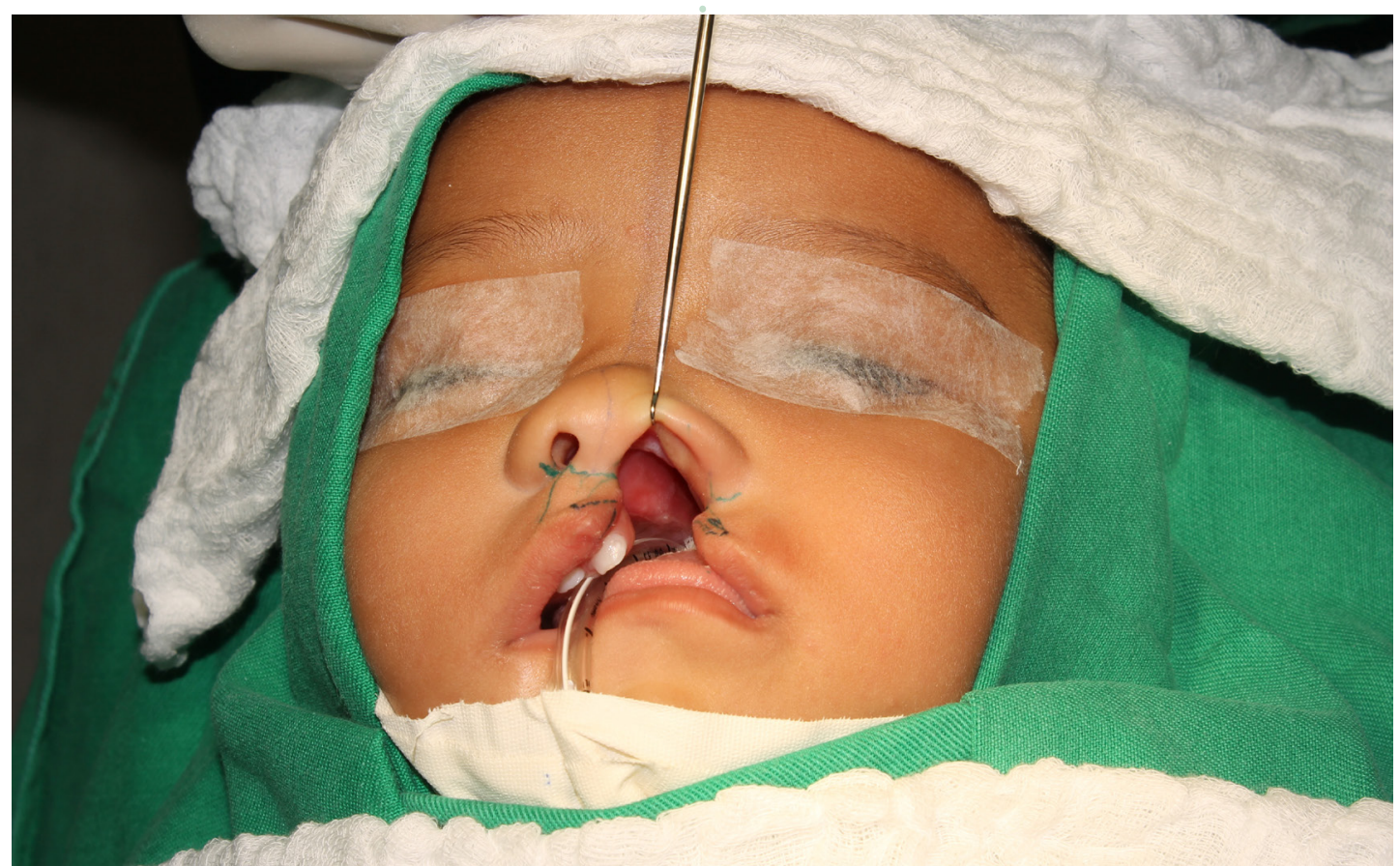

Figura 1a

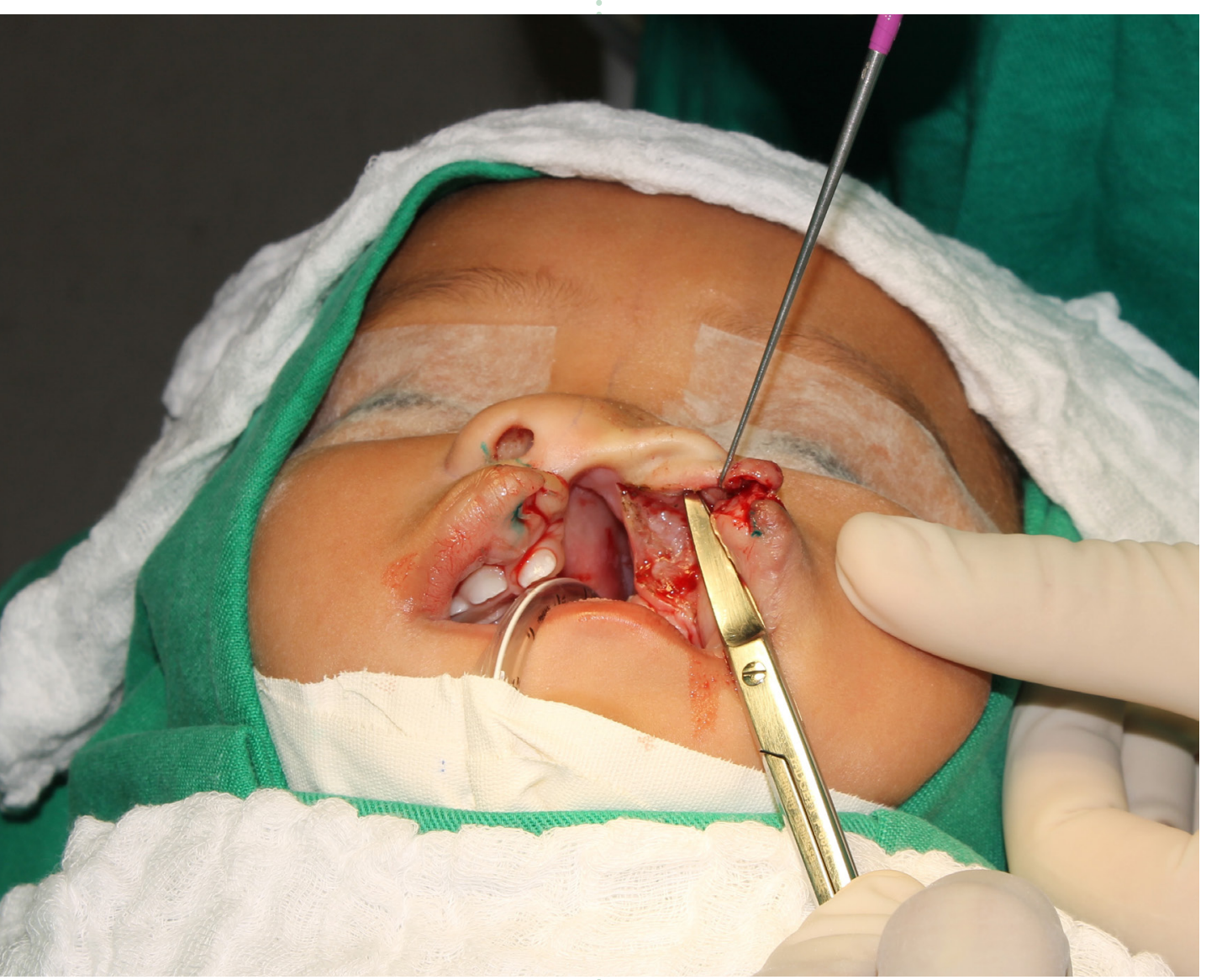

Figura $1 b$ 


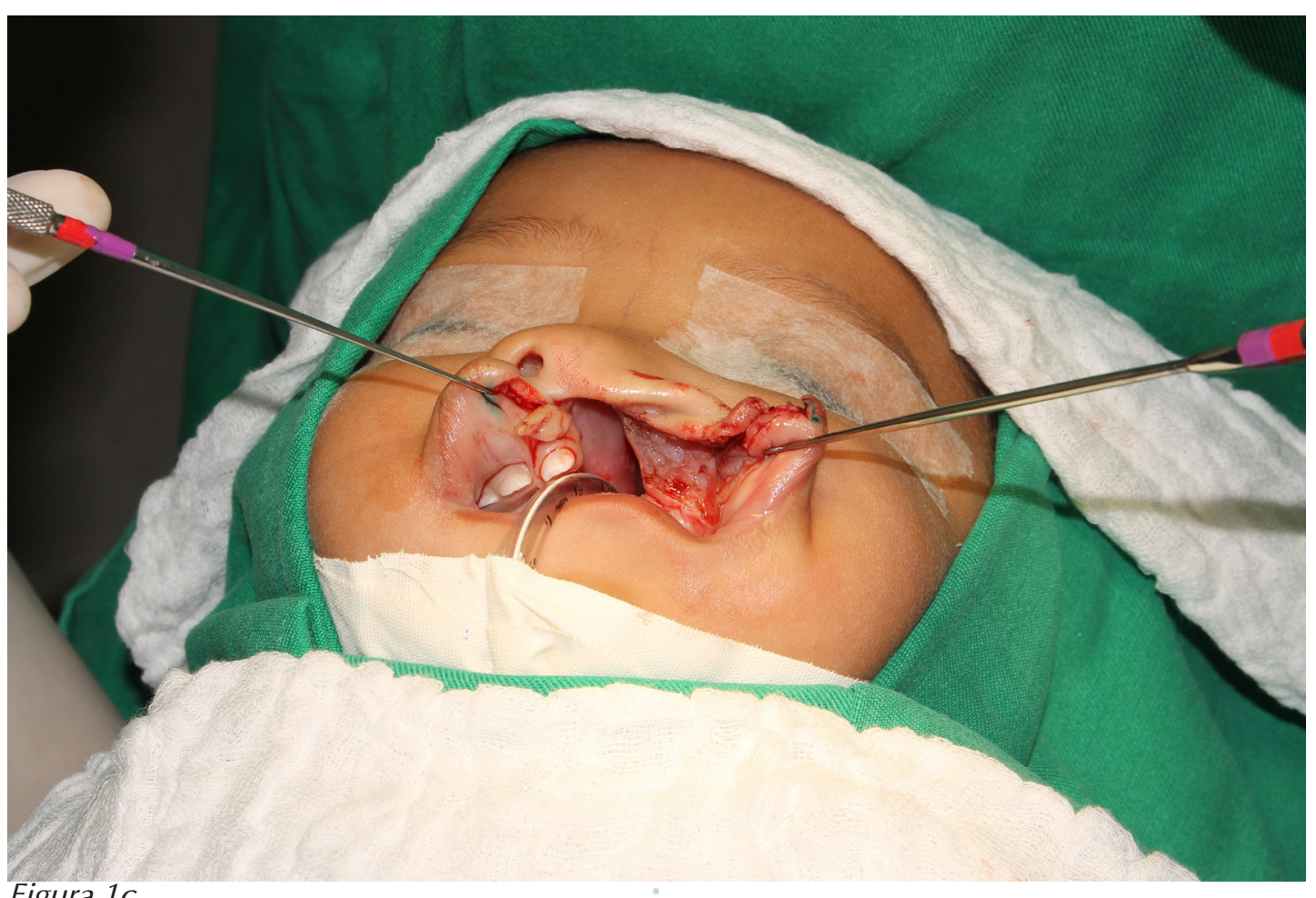

Figura 1c

reabsorvível vicryl 4-0 (Figura 2). Posteriormente realizada a suspensão das cartilagens nasais com fio de sutura vicryl 4-0. Após 30 dias de cirurgia o paciente

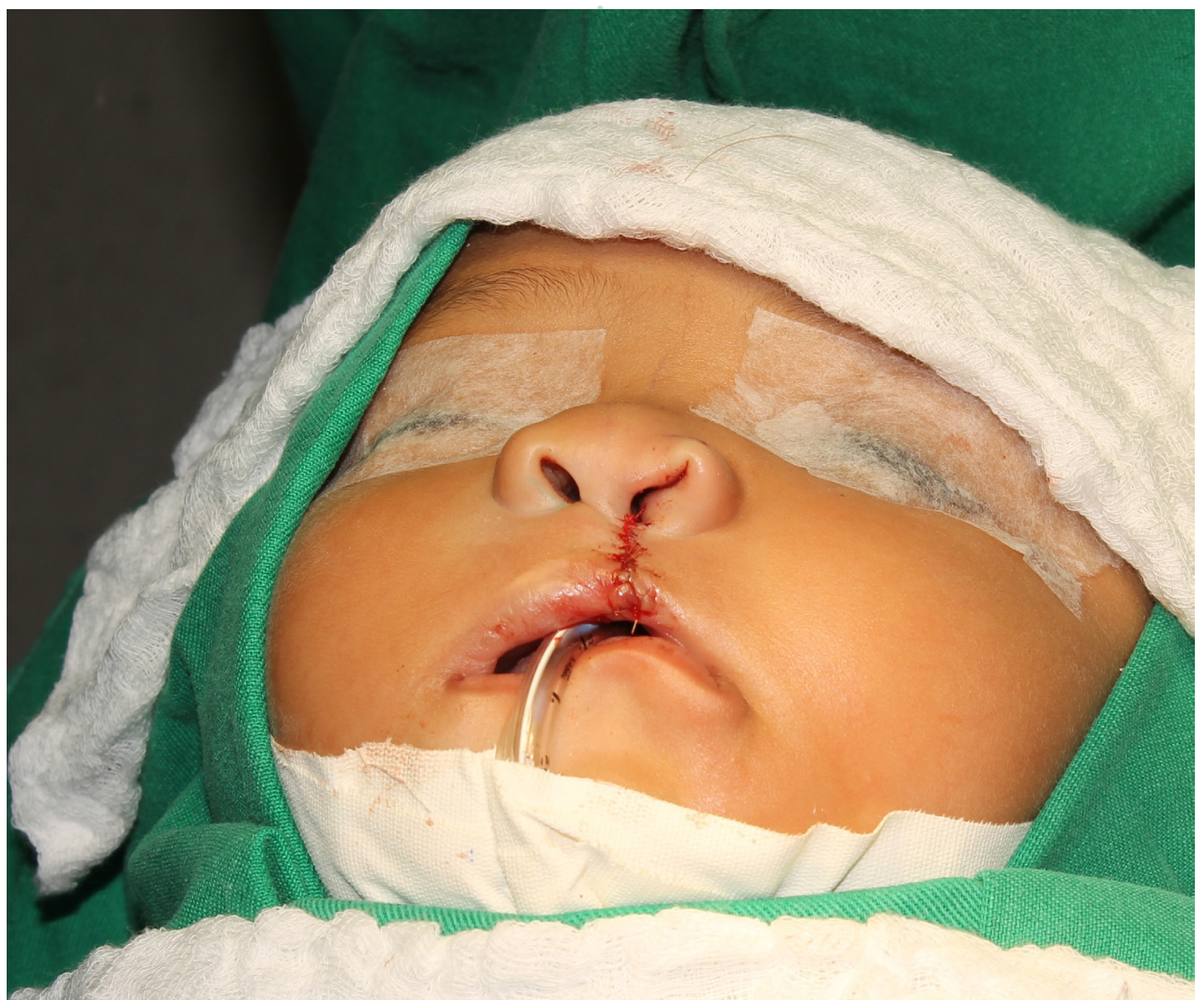

Figura 2
SALES PHH

ROCHA SS

ALBUQUERQUE AFM

CUNHA-FILHO JF

QUEILOPLASTIA

PRIMÁRIA

UNILATERAL

ATRAVÉS DA

TÉCNICA DE

FISHER

encontrava-se com ótima cicatrização da região cutânea do lábio superior, com boa simetria do filtro labial e cicatriz pouco visível (Figura 3).

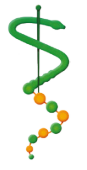


SALES PHH

ROCHA SS ALBUQUERQUE AFM CUNHA-FILHO JF

QUEILOPLASTIA PRIMÁRIA UNI LATERAL ATRAVÉS DA TÉCNICA DE FISHER

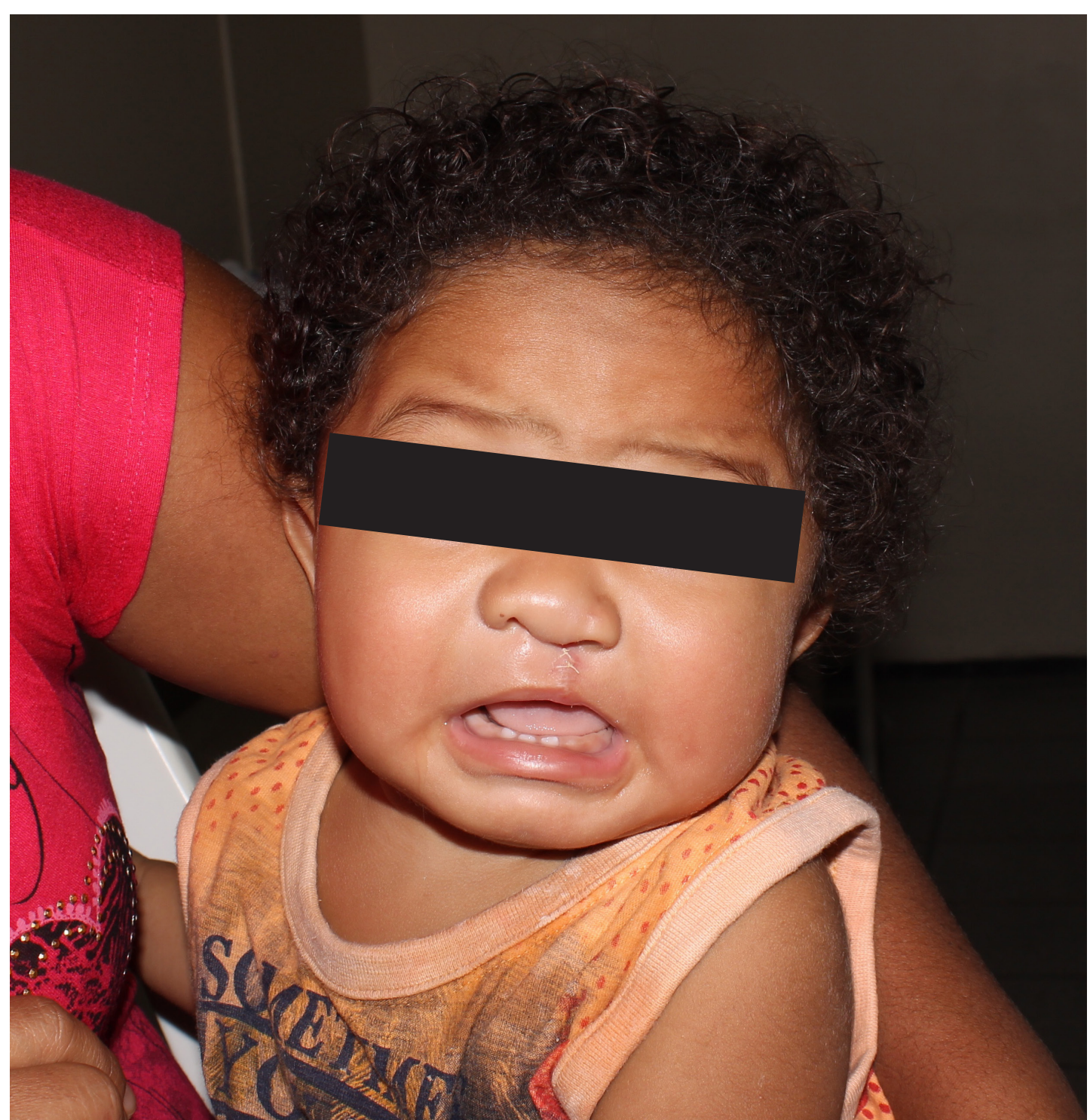

Figura 3

\section{I S C U S S ÃO}

O objetivo geral de toda a cirurgia de lábio fissurado é promover ao paciente uma estética facial adequada e uma correta função do músculo orbicular da boca e das estruturas orofaciais como um todo. Especialmente nos últimos cem anos, diversos autores têm criado e modificado técnicas cirúrgicas para reparação do lábio fissurado, com a finalidade de atingir esses objetivos de maneira ideal.

Autores como Rose e Thompson propuseram suas técnicas cirúrgicas baseadas em desenhos em linha reta. Essa técnica apresenta a facilidade de execução bem como de entendimento, entretanto a possibilidade de encurtamento do lábio por retração cicatricial torna os resultados pobres a longo prazo ${ }^{6}$.
As técnicas que utilizam desenhos geométricos são as mais populares entre os cirurgiões. Elas combinam rotações com avanços de retalhos em formas triangulares ou quadrangulares ${ }^{6}$. A técnica de Tennison utiliza princípios de retalhos triangulares. Essa técnica mostra bons resultados na aparência do vermelhão e pele do lábio e em fissuras labiais amplas ${ }^{8}$ ${ }^{9}$. É realizada uma incisão em triângulo próximo ao terço inferior do filtro do lábio, o qual é desenhado com o objetivo de alongar o lábio durante a cirurgia.

A técnica de Fisher utiliza princípios da técnica de Tennison com retalhos triangulares. É uma técnica mais recente e portanto menos utilizada pelos cirurgiões, entretanto os resultados obtidos têm sido excelentes. Essa Técnica proporciona uma cicatriz bem posicionada sobre o filtro e é 
mais fácil de ser executada do que outras técnicas geométricas, visto que é uma técnica quase que linear ${ }^{1,7}$.

Vários autores vêm descrevendo modificações de técnicas tradicionais, tentando obter um desenho mais individual, com menos incisões e melhores resultados 9 . A técnica de Millard proporcionou uma revolução à época, pois trouxe conceitos de rotação de retalho e avanço que não existiam e, por sua versatilidade e facilidade de execução, é a mais realizada nos Estados Unidos e a mais ensinada durante os programas de residência ${ }^{1,6,8}$; entretanto, como sua incisão cruza o filtro labial, a cicatriz pode gerar um efeito estético desagradável. Esse é um dos motivos que fazem com que a técnica tradicional de Millard não seja nossa primeira escolha para a cirurgia de queiloplastia unilateral.

A combinação da técnica de Reichert-Millard vem proporcionando bons resultados em comparação à tradicional, pois são realizadas menos incisões e a cicatriz fica localizada paralela ao filtro labial, deixando um aspecto mais natural ${ }^{9}$.

É extremamente importante que os cirurgiões dominem várias técnicas de reparos de lábios fissurados, pois os padrões da fissura podem variar de um paciente para outro. Pacientes com fendas labiais menores se beneficiam bem da técnica de Millard, enquanto pacientes com fissuras maiores são mais beneficiados com técnicas como as de Tennison e Fisher que tendem a alongar mais o lábio ${ }^{2,7}$.

$\mathrm{Na}$ técnica de Fisher, utiliza-se um retalho triangular imediatamente acima da linha muco-cutânea do lábio. Esse retaIho tem como função o alongamento do lábio, fazendo com que a maior parte da cicatriz fique camuflada, deixando-a em uma posição anatômica mais favorável ${ }^{7}$.

Atualmente nossa técnica de escolha para cirurgia de lábio unilateral tem sido a de Fisher, pelos motivos acima mencionados. No presente caso, o triângulo realizado próximo à linha muco-cutânea do lábio foi pequeno, visto que durante o fechamento foi observado que o lábio não necessitava de um alongamento significativo.

\section{CONSIDERAÇÕES FINA I S}

Diversas técnicas têm sido descritas para os tratamentos de fissura labial unilateral e a escolha da técnica deve passar pela avaliação criteriosa do paciente, escolha e experiência do profissional. A técnica de Fisher tem mostrado bons resultados estéticos, com cicatrizes posicionadas na crista do filtro labial e bons resultados funcionais quanto ao correto posicionamento da musculatura orbicular e do arco do cupido.
SALES PHH

ROCHA SS

ALBUQUERQUE AFM

CUNHA-FILHO JF

QUEILOPLASTIA

PRIMÁRIA

UNILATERAL

ATRAVÉS DA

tÉCNICA DE

FISHER

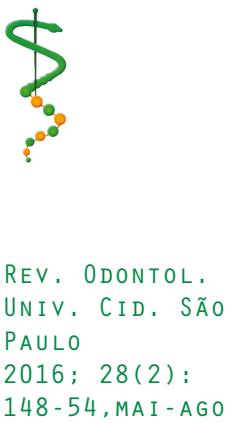


SALES PHH

ROCHA SS ALBUQUERQUE AFM CUNHA-FILHO JF

QUEILOPLASTIA PRIMÁRIA UNILATERAL ATRAVÉS DA TÉCNICA DE FISHER

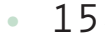

1. Freitas RS, Kurogi AS, Nasser IJG, Canan Junior LW, Busato LB. Tratamento da fissura labial unilateral pela técnica de Fisher: uma série de casos. Rev Bras Cir Craniomaxilofac 2011 14(1):2933.

2. Valente AMSL, Espinosa MM, Silva AN, De Luccia G. Caracteristicas dos pacientes submetidos a cirurgias corretivas primárias de fissuras labiopalatinas. Rev HCPA 2013 jan-mar;33(1):32-9.

3. Biazon J, Peniche AdCG. Estudo retrospectivo das complicações pós-operatórias em cirurgia primária de lábio e palato. Revista da Escola de Enfermagem da USP 2008 42(3):51925.

4. Meyer E, Seyfer A. Cleft lip repair: technical refinements for the wide cleft. Craniomaxillofacial trauma \& reconstruction 2010 Jun;3(2):81-6.
5. Meara JG, Andrews BT, Ridgway EB, Raisolsadat MA, Hiradfar M. Unilateral cleft lip and nasal repair: techniques and principles. Iranian journal of pediatrics 2011 Jun;21(2):129-38.

6. Demke JC, Tatum SA. Analysis and evolution of rotation principles in unilateral cleft lip repair. Journal of plastic, reconstructive \& aesthetic surgery : JPRAS 2011 Mar;64(3):313-8.

7. Fisher DM. Unilateral cleft lip repair: an anatomical subunit approximation technique. Plastic and reconstructive surgery 2005 Jul;116(1):61-71.

8. Zaleckas L, Linkeviciene L, Olekas J, Kutra N. The comparison of different surgical techniques used for repair of complete unilateral cleft lip. Medicina (Kaunas, Lithuania) 2011 47(2):85-90.

9. Rossell Perry PE. Combinación de la técnica de Reichert-Millard en el tratamiento de la fisura labial unilateral Acta Med Peru 2008 ene.-mar.;25(1):16-21.

Recebido em 21/07/2016

Aceito em 08/08/2016
REV. ODONTOL. UNIV, CID, SÃO PAULO: 2016; 28(2): $148-54$, MAI - AGO 